\title{
EDITORIAL
}

\section{Winners of the 2014 JA Medals for excellence}

The Journal of Antibiotics (2015) 68, 1-2; doi:10.1038/ja.2014.156

$\mathrm{T}$ he Editorial Board of The Journal of Antibiotics is happy to announce the winners of the 2014 JA Medals for review and original publications. Eligible contributions were published in the journal over the past 36 months.

The 2014 JA Medal for reviews is entitled 'Towards a new science of secondary metabolism' by Arryn Craney, Salman Ahmed and Justin Nodwell from the University of Toronto (The Journal of Antibiotics volume 66 , pages $387-400,2013) .{ }^{1}$ In this timely review, the authors report the state of our current understanding of the regulation of biosynthetic gene clusters in actinomycetes and its application in mining new natural product production and increasing production of compounds. They explain the similarities and differences in regulatory strategies that are specific to individual clusters and those that are involved in the expression of many distinct compounds. A common thread in the review is the idea of rationally exploiting these mechanisms in strain improvement and selective measures to boost natural product production. The review nicely knits together historical and recent information on natural product expression and as a result is of great importance to researchers interested in modulating the expression of compounds and fully exploiting the chemical diversity of these remarkable bacteria.

The JA Medal for an original article is awarded for a paper entitled 'Occurrence, distribution, dereplication and efficient discovery of thiazolyl peptides by sensitive-resistant pair screening' authored by a group (Sheo B Singh et al.) from Merck Laboratories in Rahway, New Jersey and the Fundación MEDIN in Granada Spain (The Journal of Antibiotics volume 66, pages 599-607, 2013). ${ }^{2}$ In this work, the authors offer a new solution to the dereplication challenge in new antibiotic discovery from natural product sources. Focusing on the thiazolyl peptide antibiotics that include such compounds as thiostrepton and thiazomycin, they report on a platform where the first step is a parallel bioassay on thiazomycin-sensitive and -resistant Staphylococcus aureus to identify extracts that have antibiotic activity vs sensitive but not resistant strains and are designated as likely containing thiazolyl antibiotics. They next developed a highly sensitive miniaturized (96-well) solid phase extraction and purification approach with analysis by high-resolution Fourier transform LCMS to dereplicate known thiazolyl antibiotics. Subsequent analysis of novel compounds identified three new members of this class of antibiotics. This work demonstrates the power of platform approaches that combine microbiological and analytical methods to narrow in on new chemical matter in antibiotic discovery.

In both the JA Medal publications for this year, the recurring theme is on novelty and innovation. This demonstrates the creativity and excitement in the microbial natural products field that are being fueled by the application of modern breakthroughs in genomics and analytical chemistry to traditional antibiotic discovery.

Gerard D Wright and Minoru Yoshida Review Editors, The Journal of Antibiotics, Japan Antibiotics Research Association, Shinagawa-ku, Tokyo, Japan

1 Craney, A., Ahmed, S., Nodwell, J. Towards a new science of secondary metabolism. J. Antibiot. 66, 387-400 (2013).

2 Singh, S. B. et al. Occurrence, distribution, dereplication and efficient discovery of thiazolyl peptides by sensitive-resistant pair screening. J. Antibiot. 66, 599-607 (2013). 


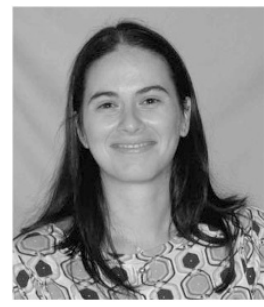

Arryn Craney received her PhD in 2012 from McMaster University under the supervision of Dr Justin Nodwell in the Department of Biochemistry and Biomedical Sciences where her studies focused on finding small molecules that alter the development and secondary metabolite production of Streptomyces. In 2012 she joined the laboratory of Dr Floyd Romesberg at the Scripps Research Institute in the Department of Chemistry as a National Science and Engineering Research Council (NSERC) postdoctoral fellow. She is currently researching the mechanisms of resistance that evolve in Staphylococcus aureus to the arylomycin family of natural products that inhibit bacterial type 1 signal peptidase, a promising antibacterial drug target. Dr Craney is also involved at the Scripps Research Institute with the postdoctoral association, the Society of Fellows, and has served as Chair of Career Development for the past 2 years and will serve as President in 2015.

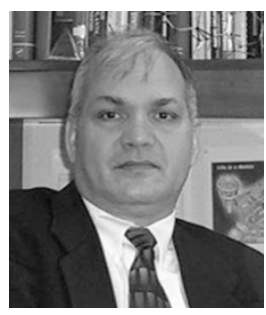

Sheo B Singh received his PhD in Natural Products Chemistry at Central Institute of Medicinal \& Aromatic Plants/Avadh University in India in 1981. After postdoctoral studies on the synthesis and biosynthesis with Prof. Karl Overton at Glasgow University followed by various research appointments to study biologically active natural products with G. Robert Pettit at Arizona State University, Dr Singh joined the Natural Products Chemistry group at Merck in 1989. He had increasing responsibilities at Merck, eventually leading the efforts of Natural Products as Director and Department Head. He retired from Merck in late 2013 as a Senior Principal Scientist and Program Lead in the Discovery Chemistry. He has discovered over 350 new biologically active natural products representing wide therapeutic areas including anticancer, cardiovascular, hypertension, antihelmintic, antiparasitic, coccidiostat, antimalarial, immunoregulant, anti-diabetics, antiviral including HIV, antifungal and antibacterial agents. He is discoverer or co-discoverer of platensimycin (antibiotic/antidiabetic), kibdelomycin (antibiotic), thiazomycin (antibiotic), nodulisporic acid (antiparasitic), apicidin (coccidiostat, antimalarial, anticancer), dolastatin-10 and -15 (anticancer) and combretastatins (anticancer). These discoveries led to more than nine preclinical development candidates including four antibiotic/antifungal agents. A natural product lead from these discoveries led to an analog, which was conjugated with an ADC to an US Food and Drug Administration-approved drug, brentuximab vedotin (ADCETRIS). Two products, OXI4503 and ZYBreSTAT, are in phase I/II/III human trials for treatment of cancer. Dr Singh has published over 220 papers, 30 reviews and chapters, 49 patents and delivered hundreds of lectures. He serves on the Editorial Boards of Journal of Natural Products, Journal of Antibiotics and Natural Product Reports. Currently he is an Independent Pharmaceutical Consultant and is President, Principal and Owner of SBS Pharma Consulting, LLC. 